This item was submitted to Loughborough's Research Repository by the author.

Items in Figshare are protected by copyright, with all rights reserved, unless otherwise indicated.

\title{
Identification of formation-stages in a polymeric foam customised by sonication via electrical resistivity measurements
}

PLEASE CITE THE PUBLISHED VERSION

http://dx.doi.org/10.1007/s10965-008-9249-4

\section{PUBLISHER}

(C) Springer

VERSION

AM (Accepted Manuscript)

\section{PUBLISHER STATEMENT}

This work is made available according to the conditions of the Creative Commons Attribution-NonCommercialNoDerivatives 4.0 International (CC BY-NC-ND 4.0) licence. Full details of this licence are available at: https://creativecommons.org/licenses/by-nc-nd/4.0/

\section{LICENCE}

CC BY-NC-ND 4.0

\section{REPOSITORY RECORD}

Torres-Sanchez, Carmen, and Jonathan R. Corney. 2019. "Identification of Formation-stages in a Polymeric Foam Customised by Sonication via Electrical Resistivity Measurements”. figshare. https://hdl.handle.net/2134/22636. 


\section{Editorial Manager(tm) for Journal of Polymer Research}

Manuscript Draft

Manuscript Number:

Title: Identification of formation stages in a polymeric foam customised by sonication via electrical resistivity measurements

Article Type: Manuscript

Section/Category:

Keywords: ultrasonic irradiation; polymerization stages; electrical resistivity; cavitation

Corresponding Author: Dr Carmen Torres, PhD

Corresponding Author's Institution: University of Strathclyde

First Author: Carmen Torres, PhD

Order of Authors: Carmen Torres, PhD; Carmen Torres, PhD; Jonathan R Corney, Professor

Manuscript Region of Origin:

Abstract: The polymerisation reactions associated with foam formation have distinct stages (i.e. nucleation, growth, packing, stiffening, solidification) some of which are known to be more sensitive to external inputs than others. Consequently, precise detection of the start and end points of each of the polymerisation stages would enable the fine control of material properties such as porosity. The development of such process control can only be pursued if those sensitive stages can be clearly distinguished during the manufacture process. This paper reports how an electrical resistivity tracking method was used to assess the differences in the foaming processes when ultrasound was irradiated to polymeric melts undergoing foaming. The electrical resistivity tracking method is also appraised with regard to its suitability to accurately identify the formation stages in the foam, as described by other authors. 



\title{
Identification of formation stages in a polymeric foam customised by sonication via electrical resistivity measurements
}

\author{
C. Torres-Sánchez and J. Corney \\ DMEM, University of Strathclyde, James Weir Building, 75 Montrose Street, \\ Glasgow, G1 1XJ, United Kingdom \\ Phone: +44 1415482091 \\ Fax: +44 1415527986 \\ Email: carmen.torres@strath.ac.uk
}

\begin{abstract}
The polymerisation reactions associated with foam formation have distinct stages (i.e. nucleation, growth, packing, stiffening, solidification) some of which are known to be more sensitive to external inputs than others. Consequently, precise detection of the start and end points of each of the polymerisation stages would enable the fine control of material properties such as porosity. The development of such process control can only be pursued if those sensitive stages can be clearly distinguished during the manufacture process. This paper reports how an electrical resistivity tracking method was used to assess the differences in the foaming processes when ultrasound was irradiated to polymeric melts undergoing foaming. The electrical resistivity tracking method is also appraised with regard to its suitability to accurately identify the formation stages in the foam, as described by other authors.
\end{abstract}

Keywords: ultrasonic irradiation; polymerization stages; electrical resistivity; cavitation

\section{Introduction}

The cellular structure of a polymeric foam determines many of its functional properties. Mechanical, thermal, acoustic properties of a solid foam are intrinsically determined by the architecture of its microstructure (i.e. the porosity distribution within the volume). Indeed, the large range of applications (in a myriad of fields: biomaterials, tissue engineering, structural mechanics, etc) for polymeric foam materials has arisen because of their versatility, which makes them an exceptional design material [1]. The challenge is to engineer processes that allow the cellular structure of foams to be easily designed and manufactured for specific functions. 
In this context it is well established that some polymerisation reactions are affected by external inputs (e.g. acoustic irradiation [2-4]) or additives (e.g. catalysts, surfactants, blowing agents, etc), which influence the cross-linking processes (e.g. enhance blending [5], accelerate gelation [6], disperse fillers or particles [7], etc). These agents allow the final structure of the solid polymer foams to be tailored creating permanent alterations in the foams' structure. However, it is also well known that the polymerisation reactions associated with foam formation have distinct stages (i.e. nucleation, growth, packing, stiffening, solidification) some of which are known to be more sensitive to external inputs than others. Consequently, monitoring the reaction in order to detect the start, transition or end point of those sensitive phases is necessary to allow the optimisation of the final properties of a polymer foam.

However, any method used to do this used needs to be largely non-intrusive because the fast growing system could become unstable and, in the extreme case, collapse.

Literature describes various methods of non-intrusive monitoring and control of polymerisation reactions. For example, the use of temperature measurements [8] to detect the end of the reaction (i.e. post peak temperature) are well established. More recently, calorimetric measurements [9] have been used for evaluating the details of the curing process. Other researchers have reported the use of non-invasive spectroscopy, FT Raman [10] and near infrared spectroscopy [11], ultrasound velocity measurements [12], and dielectric monitoring [13]. However these methods either offer only limited detection of the specific stages of formation or required very expensive equipment.

Continuous monitoring of the variation in electrical properties throughout the polymeric reaction is proven as a reliable and low cost method that allows the detection of sensitive stages and, therefore, provides instant feedback on how the 'agent' is affecting the reaction. One of the outstanding benefits that electrical conductivity offers is that it permits the internal state of the foam to be monitored, which makes this a particularly valuable technique in processes where foams are opaque and/or visual inspection is not an option [14] (e.g. continuous monitoring of draining rates and stability of foams [15]). For example, conductivity measurements have been used for online monitoring of emulsion polymerisations [16], where the stability of particles after the addition of a surfactant was assessed. Dielectric measurement, coupled with more traditional techniques such as 
viscosity [17] to measure the curing degree in foaming-controlled additives, and temperature [18] to monitor the particle population available for coagulation, still remains as one of the most promising techniques for non-destructive testing and diagnostic [19].

This paper's objective is twofold:

1. Report the feasibility of using electrical resistivity properties as a reliable method of both monitoring the overall progress and detecting distinct stages in a polymeric melt undergoing foaming.

2. Demonstrate how this method (i.e. electrical resistivity measurement) can be used to determine the effects of ultrasound on a polymeric foaming process.

The rest of the paper is structured as follows: the background section gives an overview of the foaming process, the effects of ultrasound on foaming polymers and the principles of electrical resistivity monitoring. Section 3 describes the experimental investigation, and section 4 presents the results. In the discussion, section 5, a theoretical rationale for the experimental results is shown before conclusions are drawn in section 6 .

\section{Background}

\section{Stages of polymeric foam formation}

Foam is the dispersion of a gas in a polymeric liquid, which creates a characteristic structure when the matrix solidifies. Once cured, the foam consists of individual cells, or pores, the walls of which have completely polymerised and solidified to form a skeletal structure. The cells can be open with interconnected cells having a thin membrane between the skeletal ribs, or closed with separate cells which are often non permeable and resistant to moisture and oil, insulating against heat and cold and absorbent of impacts or vibrations.

Polyurethane foam (PU) is an example of solid foam which is made by the polymerisation of a monomer mixture (polyol and isocyanate) after the addition of a catalyst [20]. The urethane group formed has robust mechanical and chemical properties that have made this polymer one of the most widely used in industry over the past seventy years. The 
polyurethane foam produced for the purpose of this study is close-celled and distilled water was used as a chemical blowing agent. The water diffuses across the holes formed between the chains of polyurethane reacting simultaneously with the isocyanate groups at the end of the chains, causing the reticulation (or cross-linking) of the polymer, and forming a semi-rigid solid. The gas product, carbon dioxide, acts as a blowing agent and fills up the cavities forming a polymeric foam once the matrix has fully solidified.

A qualitative description of the foaming process using a chemical blowing agent can be described in terms of the following five characteristic stages [21-25] and the events that take place in each of them (Table 1):

1. Cream stage: Bubble nucleation occurs upon the addition of the catalyst (i.e. water). The reaction produces carbon dioxide gas, $\mathrm{CO}_{2}$, which acts as the blowing agent and gives a cloudy, creamy appearance to the mixture.

2. Rise stage: After nucleation, the polymeric mass begins to expand in volume. Foaming occurs at constant pressure but with variable volume (i.e. free expansion in an open vessel). During this stage the liquid foam is a metastable system that evolves dynamically due to two processes: foam drainage (liquid flows through the interstitial volume between bubbles) and foam coarsening (gas exchange between bubbles). Coarsening accelerates drainage and consequently foam breakdown.

3. Packing stage: The chemical blowing agent raises the polymeric melt until a maximum height is reached. Then, the exothermic energy of the reaction is used for the creation of links between monomers, cross-linking, creating long chains that form the polymer, entangled units that will form cells. In the case of open-pore foams, the process develops further and the pressure inside each bubble is sufficient to provoke rupture of cell walls that separate bubbles, creating open channels.

4. Gelation stage: At this point, the final structure of the foam is reached. The rigidity of the fluid is high enough to consider the bubbles as cells. Bubble size becomes fixed and there is no longer the possibility of the bubbles expanding or collapsing, as the increasing viscosity of the plastic makes the walls stiff and strong against shear forces. The gel point is typically obtained experimentally by periodically moving a thermocouple during the reaction and noting the temperature and time at which the mixture seems solid.

Conversion rate (the ratio of products to reactants) at that point is considered by some authors $[8,21]$ to be approximately 0.5 . 
5. Solidification stage: Finally, when all the polymeric mass has gelled, the final structure is obtained. The end of the reaction happens when the conversion rate reaches unity. Cross-linking finishes and foam starts a curing period where cells become fully solidified.

\section{Effects of ultrasonic irradiation in the manufacture of porosity graded polymeric foams}

Bubble dynamics play an important role in the foaming process of polymeric melts, and recent work has established that ultrasound applied to the system can influence the dynamics of the process [26-28]. These sonication effects affect both bubble growth and nucleation rates in foams since both are strongly influenced by the concentration of dissolved gas in the resin (in other words, the saturation level determines the gas pressure and hence the driving force for bubble growth).

The work reported in $[29,33]$ demonstrated that bubble growth can be controlled by ultrasonic irradiation, which when applied at those acoustically sensitive stages of the physical-chemical reaction, can modify (accelerate or retard with acoustic parameters such as power, frequency, etc) the time span, or rate, of the 'packing' and 'gelation' stages and, therefore has an influence on the size of the pores, their wall thickness or their orientation. These changes are manifested in the final cellular structure once the foam has solidified.

Particularly important in the context of foams, and other high viscosity fluids, is the ability of ultrasound to produce an increase in mass transport due to diffusion variation [29], facilitating in this way diffusion and mixing of constituents. Essentially, sound affects the viscosity of fluids significantly (usually decreasing their viscosity) [28, 30], so acoustic radiation reduces the diffusion boundary layer, increasing the concentration gradient and, to a certain extent, may also increase the diffusion coefficient. Since viscosity is inversely proportional to the diffusion coefficient, the latter will increase in sound fields. Convection also decreases the thickness of the mass transfer boundary layer, i.e. the wall of the pore. Hence, an increase in the membrane transport is due to reduced wall thickness. In addition, ultrasound creates stresses that disrupt the normal configuration (shape, wall thickness) of pores, and thus increases the membrane 
permeability towards gas/vapour (the blowing agent). However, if the intensity (i.e. pressure amplitude) is too high, ultrasound can go above the threshold and become transient, provoking shockwaves that can rupture cell walls.

In summary: controlled ultrasonic irradiation affects convective mass transfer during foaming, especially during rising and packing stages, and enhances diffusion of the blowing agent (i.e. $\mathrm{CO}_{2}$ gas) from bubble to bubble in the packing and gelation stages [31].

\section{Electrical resistivity principles}

Electrical resistivity of a foam indicates its ability to impede the flow of electrical current through the substance. The hypothesis for the electrical resistivity monitoring is that its variation during the reaction reflects the state of the process (e.g. liquid, rising, crosslinking, solid, etc). In the liquid state, resistivity will be very low (i.e. high electrical conductivity due to the dissolved species). However, during cross-linking, the electrical resistivity will increase due to pore formation, producing cavities that will dry up by the end of the reaction (Figure 1). This situation will produce a very high value of electrical resistivity readings, where in addition to the material properties and the foam drainage, the conductive path will increase its tortuosity. Consequently, it can be hypothesised that the resistivity of a foam depends on the conductivity of the foam components and the amount of liquid filling the pore architecture, which varies depending on the stage of the reaction.

Mathematical relationships between electrical properties and mechanical properties have been widely reported in the literature where researchers have been motivated by the desire to create non-destructive testing methods [32-34]. In the same way, electrical resistivity of a foam can be measured by applying a voltage between two probes which are in intimate contact with the material and measuring the response voltage, by the following relationship:

$$
R_{x}=\rho \cdot \frac{L}{A}
$$

Where $R_{x}$ represents the resistivity, $\rho$ the dielectric constant of the material, $L$, the fixed distance between the measuring probes, and A cross-sectional area of the conductor (i.e. the liquid component of the foam between the two probes). 


\section{Experimental investigation}

Because only some phases of the foaming process are sensitive to sonication [29], it is important to enable a systematic monitoring of the progress of the reaction, so the start and end points of each stage can be clearly identified and, therefore, the ultrasound irradiation applied to the samples in a controlled manner.

\section{Methodology}

The electrical resistivity variation was measured during the formation of polyurethane foam samples in a temperature controlled water bath both with and without ultrasound irradiation. When samples were sonicated, a range of frequencies and powers were systematically explored. Details of the arrangement of ultrasonic sources/receivers for the experimental sessions and sensors within the water bath can be found elsewhere [35].

A description of the procedure used for the formation, and in some cases irradiation, of the foams is shown in Figure 2:

The polymers used in this study (Dow Pro Series polyurethane Foam, Dow Europe $\mathrm{GmbH}$, Switzerland, RS 202-2636), were degassed from the blowing components (i.e. methane, ether, isobutane) by dissolving in pure acetone. Acetone also assisted in removing moisture. It was important that these gases were removed from the mixture to allow control of the amount of initially dissolved gas. In all cases, the diisocyanate content in the mixture was rectified to have a fixed $40 \%$. The relation PU-Acetone used was $50 / 50 \%$ vol. The container with the caramel coloured liquid was placed inside a water bath and both thermocouple and conductivity probes inserted at this time (to obtain readings of conductivity and temperature when the water, catalyst for the reaction, was added).

Although the electrical resistivity is weakly dependant on temperature [36] for the range of working temperatures and pressures (i.e. open vessel) used here, the water bath was employed to ensure the temperature of the environment could be controlled independently of the effects of ultrasound, and the temperature gradient due to the polyurethane exothermic reaction could therefore be diminished. The bath temperature was set at $323 \mathrm{~K}$ 
and controlled within $+/-1 \mathrm{~K}$. A higher temperature was not desired as it would increase the probability of side reactions [8].

The polyurethane foaming reaction was initialised by the addition of warm distilled water, which acted as a catalyst for the reaction and chemical blowing agent for the foaming process. This predetermined amount of distilled water $\left(20 \% \mathrm{vol} \mathrm{H}_{2} \mathrm{O}\right.$ per ml of diisocyanate present in the mixture) was added drop-wise by syringe. The solution then turned into a milky brown colour due to the suspension of water/acetone droplets, which were perfectly miscible. The mixture was stirred for 70 seconds using a standard procedure and minimising air intake into the mixture. The addition of water simultaneously induced the generation of urethane and the formation of $\mathrm{CO}_{2 \text { (gas) }}(\mathrm{Cream}$ Stage).

Rapid cell expansion occurred at the beginning of the reaction due to the high rate of $\mathrm{CO}_{2 \text { (gas) }}$ production (Rising Stage). When ultrasonic irradiation was applied to the samples, it commenced at this point. 5-6 minutes after the reaction started, the stable height of the foam was reached. At the end of the Rising Stage, the production of $\mathrm{CO}_{2}$ (gas) was considered to be finished. This event happened at the, so called, 'gelation point' (Gelation Stage). The final structure of the foam (cells walls bounding voids) was obtained. Bubbles could neither expand nor collapse. The overall reaction continued strengthening the walls: the polymerisation of urethane and its cross-linking (Solidification Stage). Ultrasonic irradiation, if any, was stopped at this point. After 10-12 minutes from the start, the foam became a solid and not sticky-to-the-touch. The temperatures dropped and followed any changes in the water bath. Samples were kept inside of the bath after the reaction finished for an extra 30 minutes until the foam had a rigid complexion. This was done to avoid sudden changes of temperature that could provoke collapse of the pores due to the migration of trapped water vapour and $\mathrm{CO}_{2(\mathrm{gas})}$. Finally, samples were placed in a warm, humidity-controlled closet for 24 hours until they were fully cured.

All mixtures were sonicated in an open-vessel container to avoid the build up of the internal pressure due to the water vapour and gases generated by the reaction that could provoke unwanted implosion of bubbles. Pairs of experiments were carried out to test the repeatability of the foaming process. The foams were irradiated for 20 minutes in a duty 
cycle of $2 \mathrm{~min}$ on/1min off, starting after adding the distilled water. The level and frequency of this cyclic irradiation was established by initial experimentation [35] and was sufficient to induce changes in the foam structure without causing collapse.

\section{Experimental rig}

Preliminary tests showed that the mixture's electrical resistivity was greater than $10^{8}$ $\Omega \mathrm{cm}$. A lower value $\left(\sim 10^{3} \Omega \mathrm{cm}\right)$ was only measured when the liquid reactants were tested and, as soon as the catalyst was added and the reaction started, the electrical resistivity increased by several orders of magnitude. Because of this steep change in resistivity, the standard measurement equipment could not be used and a bespoke resistivity tracking cell was designed and assembled in-house.

The experimental rig shown below measured the electric resistivity of the foams by using a two-probe resistivity measurement method. Data was recorded using an acquisition system for each sample. The circuit diagram is presented in Figure 3. In this method, the resistance offered by the foam $\left(R_{x}\right)$ to the excitation voltage applied (Base voltage, $V_{B S}$, 2.5 volts DC) across the two measuring probes (terminals) immersed in the container where the reaction happened, was measured. To do that, a potential drop produced by the foam resistance was measured after amplification of the signal at the instrumentation amplifier (Op-Amp). The output from the logic function in the Op-Amp was collected by the Data Acquisition Card (DAQ, DS1M12 StingRay, USB Instruments ${ }^{\text {TM }}$, EasySync Ltd., UK) as shown in Figure $3 b$.

The dimensions of the copper probes used were $2 \mathrm{~mm}$ in diameter and $7 \mathrm{~cm}$ in length, with a fix interval of $39 \mathrm{~mm}$ between probes. Since the separation between these two surfaces was constant (fixed electrode spacing) and the applied excitation voltage $\left(\mathrm{V}_{\mathrm{BS}}\right)$ is constant, the electrical resistance of this volume of foam was determined by measuring the output potential difference. The relationship between the output voltage and the foam resistance can be expressed as a function of the system parameters (i.e. reference resistors $(\mathrm{R})$, voltage base $\left(\mathrm{V}_{\mathrm{BS}}\right)$, gain $\left.(\mathrm{G})\right)$ :

$$
R_{x}=\frac{V_{o} \cdot R}{G \cdot V_{B S}-V_{o}}=\frac{V_{o} \cdot 510 k \Omega}{12.419-V_{o}}
$$

Equation (2): Relationship between foam resistance $\left(\mathrm{R}_{\mathrm{x}}\right)$ value and output voltage $\left(\mathrm{V}_{0}\right)$ 


\section{Results}

This section summarises the results from the experiments to assess the variation in resistivity during the foaming process. Firstly, a correlation between the resistivity variations with the formation stages in the foam is established; then the influence of acoustic pressure on the reaction rate for the polymeric samples is presented.

Changes in electrical resistivity and temperature were logged and recorded, and both curves simultaneously changed at the point when the reaction was finished: temperature dropped and from that point onwards, it tended towards the bath temperature. As expected [8], the maximum temperature recorded coincided with the end of the reaction. Electrical resistivity became asymptotic at that point. The variations in temperature during the foam formation stages were difficult to assess due to the small range of values that were recorded (i.e. small difference between maximum value and bath temperature) and the large "inertia" that temperature readings had due to the high specific heat value of the water surrounding the reaction container in the bath. On the contrary, resistivity readings varied in a broader range, therefore it was easier to identify any variations and results could be interpreted with less ambiguity. Signals would vary in a broader range ( $\sim 200 \mathrm{mV}$ in average) from start to end of reaction compared to the signal reading for temperature, which varied only around $25 \mathrm{mV}$.

Using the relationship established for the applied voltage, $\mathrm{V}_{\mathrm{BS}}(\mathrm{mV})$, in equation (2), the value for the electrical resistivity, $\mathrm{R}_{\mathrm{x}}$, was obtained. A representative example of the electrical resistance data collected is shown in Figure 4a.

The arrows on the curve (Figure 4a) locate the phenomena occurring to the polymer as it changes from a liquid mixture to a solid cellular structure. During the foaming of the polymeric melt, the wetted surface on the measuring probes varied with time. In a first instant (1), the wetted area on the probes corresponded to the liquid mixture volume held in the container, and the resistivity value of the solution (i.e. monomers, acetone and water). When the foam was growing, the wetted surface increased with the foam's height in the vessel (2). In the proximity of the final height (3), the liquid foam was a metastable system (i.e. being an unstable and transitory but relatively long-lived state) that evolved dynamically: during expansion, cell wall thinning might have occurred, provoking temporary rupture of these (i.e. partial collapses and oscillations around its 
final height). A characteristic step change in resistance (a 'double belly' profile) was repeatedly observed.

In later events, after the final height was reached (3), the overall drying of the foam takes place. The non-reactant liquids percolated (e.g. water) to the bottom of the vessel or, eventually, evaporated (e.g. acetone). The gas produced by the chemical reaction between monomers occupied the spaces created by the cavities or escaped to the atmosphere.

During the 'packing' stage (4-5), the cross-linking process increased the tortuosity of the effective path length between the probes, (i.e. the material formed cavities that were interconnected among them by 'necks', L in equation (1)), and the wetted cross-sectional area that conducts (i.e. area wetted by liquid between the copper pens, A in equation (1)) decreased due to the drainage process of the foam. These phenomena produced an increasing rate of electrical resistivity over time that could be fitted to a logarithmic curve (Figure 4b).

At point (6), the foam surface was non-sticky and had a springy surface. After that point, temperature did not show any variation and the bath temperature was read. Reaction was considered terminated (7). Logging was stopped (8). The total reaction time for the samples to become fully solid polyurethane foams was 10-14 min, in average.

Using the resistivity variation with time, these values could be fitted to a curve of the form ' $y=a \ln (x)+b$ '. Then, the ' $a$ ' coefficient of that function was used to compare different sets of experiments in order to study the influence of ultrasonic irradiation magnitude on the development of the reaction (Figure $4 b$ ). When the ' $a$ ' coefficients from 26 samples irradiated at $20 \mathrm{kHz}$ were plotted against the acoustic pressure values (measured with a hydrophone) that they were subject to, a clear incremental trend can be observed (Figure 5). In other words, the larger the value of 'a', the higher the reaction rate was and the quicker the foam structure reached its gelation point.

\section{Discussion}

\section{Comparison of experimental results with literature}

This section offers an interpretation of the raw data in terms of variation in the electrical resistivity. A comparison between the variations observed and the stages reported in the literature are presented. 
Table 2 below summarises the information provided by the electrical resistivity measurements and maps each stage described in the introductory sections to events that the foam suffers during formation at the macro and microscopic level.

Results indicate that the changes in the electrical resistivity during the foaming process can be directly matched to the start/end point of the formation stages in the polymeric foam (cream stage, rising stage, gelation stage and, finally, solidification stage at the end of the reaction).

\section{Influence of ultrasound in the 'packing' and 'gelation' stages}

The electrical resistivity monitoring procedure has demonstrated its suitability to offer punctual information about the progress of the reaction. Particularly it has offered valuable information on the progress of the 'packing' and 'gelation' stages, known to be sensitive stages to ultrasonic irradiation. Data from Figure 5 show that the 'a' coefficient increased with increasing acoustic magnitude, confirming the role of ultrasound as mechanical gelation rate enhancer agent. Hence, a quantification of the ultrasonic irradiation onto the final solid sample could be established for a finer adjustment of functionalities in the foam (e.g. pore size and porosity distribution). Along with that, this monitoring technique allows the detection of abnormalities during the cross-linking process, partial collapses during rising time or premature coagulation of the matrix can also be detected in the manufacturing processes of polymeric foams.

This electrical resistivity measuring technique allows the tracking and identification of stages of foam formation 'sensitive to ultrasonic irradiation'. However, it also has limitations. One of most obvious ones is that the local changes of porosity cannot be detected, because only the bulk resistivity on the material is measured. A different approach needs to be followed when aiming the measurement of anisotropy in the foaming polymer by conductivity variations. In addition to this, the pens have to be in intimate contact with the foam at early stages for satisfactory results and these pens have to be pre-tempered (i.e. at the same temperature as the reaction) in order to avoid instabilities when the pens are inserted. It is evident that this method evaluates electrical conductivity variations only for bulk volumes. 


\section{Conclusion and future work}

Because only some phases of the foaming process are sensitive to sonication, it is desirable to monitor the progress of the reaction so the start and end points of each stage can be identified. In this study, a correlation of resistivity changes with the start/end of the different stages of the polymeric reaction has been shown (i.e. progression through pre-gelation, gelation and post-gelation stages). The advantages that electrical properties offer for this kind of manufacturing process is that not only 'gel point' can be detected, but also reaction rate (i.e. how fast get point is reached) can be measured. Therefore, it can be concluded that this method allows the identification of polymerisation stages, the detection of those regions sensitive to an external agent (i.e. ultrasound) and, in consequence, permits the refinement of polymeric foams for specific functional designs. Future work will focus on increasing the accuracy of the measuring device (decrease the noise) in order to make it more responsive to small changes in conductivity. With the aid of dynamic imaging (e.g. micro-CT scanners), the authors hope to establish a correlation between the resistivity changes and 3D images of the foam as it is expanding. Another research avenue opens up when different polymeric foams are considered. The electric resistivity ranges during the manufacture of those structures need to be studied to assess whether this strategy can be used as well as tracking method for the pre-gel, gel and postgelation stages in those foams.

\section{REFERENCES}

1. L. J. Gibson, M. F. Ashby, Cellular solids. Structure and properties. 2nd. ed. Cambridge University Press. 1997.

2. K. S. Suslick, G. J. Price, Applications of ultrasound to materials chemistry, Annual Review of Materials Science 29 (1999): 295-326.

3. I. Masselin, X. Chasseray, L. Durand-Bourlier, J. M. Laine, P. Y. Syzaret, D. Lemordant, Effect of sonication on polymeric membranes, Journal of Membrane Science 181 (2001): 213-220.

4. G. J. Price, A. J. White, A. A. Clifton, The effect of high-intensity ultrasound on solid polymers, Polymer 36 (1995): 4919-4925.

5. G. S. Chen, S. Y. Guo, H. L. Li, Ultrasonic improvement of the compatibility and rheological behavior of high-density polyethylene/polystyrene blends, Journal of Applied Polymer Science 86 (2002): 23-32.

6. N. Tonanon, A. Siyasukh, W. Tanthapanichakoon, H. Nishihara, S. R. Mukai, H. Tamon, Improvement of mesoporosity of carbon cryogels by ultrasonic irradiation, Carbon 43 (2005): 525-531. 
7. Y. Z. Chen, H. L. Li, Effect of ultrasound on the morphology and properties of polypropylene/inorganic filler composites, Journal of Applied Polymer Science 97 (2005): 1553-1560.

8. S. D. Lipshitz, C. W. Macosko, Kinetics and energetics of a fast polyurethane cure, Journal of Applied Polymer Science 21 (1977): 2029-2039.

9. J. R. Vega, L. M. Gugliotta, G. R. Meira, Emulsion copolymerization of acrylonitrile and butadiene. Semibatch strategies for controlling molecular structure on the basis of calorimetric measurements, Polymer Reaction Engineering 10 (2002): 59-82.

10. C. Wang, T. J. Vickers, J. B. Schlenoff, C. K. Mann, Insitu Monitoring of Emulsion Polymerization Using Fiberoptic Raman-Spectroscopy, Applied Spectroscopy 46 (1992): 1729-1731. 11. R. A. M. Vieira, C. Sayer, E. L. Lima, J. C. Pinto, In-line and in situ monitoring of semi-batch emulsion copolymerizations using near-infrared spectroscopy, Journal of Applied Polymer Science 84 (2002): 2670-2682.

12. L. Cavin, T. Meyer, A. Renken, On-line conversion monitoring through ultrasound velocity measurements in bulk styrene polymerization in a recycle reactor - Part I: Experimental validation, Polymer Reaction Engineering 8 (2000): 201-223.

13. A. J. Bur, S. C. Roth, M. McBrearty, In-line dielectric monitoring during extrusion of filled polymers, Review of Scientific Instruments 73 (2002): 2097-2102.

14. A. Phianmongkhol, J. Varley, A multi point conductivity measurement system for characterisation of protein foams, Colloids and Surfaces B: Biointerfaces 12 (1999): 247-259.

15. R. Lemlich, A theory for the limiting conductivity of polyhedral foam at low density, Journal of Colloid and Interface Science 64 (1978): 107-110.

16. C. Graillat, A. Santos, J. C. Pinto, T. F. McKenna, On-line monitoring of emulsion polymerisation using conductivity measurements, Macromolecular Symposia 206 (2004): 433-442.

17. L. Vandenbossche, L. Dupre, J. Melkebeek, On-line cure monitoring of polyurethane foams by dielectrometric viscosity measurements, International Journal of Applied Electromagnetics and Mechanics 25 (2007): 589-593.

18. A. F. Santos, E. L. Lima, J. C. Pinto, C. Graillat, T. McKenna, Online monitoring of the evolution of the number of particles in emulsion polymerization by conductivity measurements. I. Model formulation, Journal of Applied Polymer Science 90 (2003): 1213-1226.

19. J. Mijovic, J. Kenny, A. Maffezzoli, A. Trivisano, F. Bellucci, L. Nicolais, The principles of dielectric measurements for in situ monitoring of composite processing, Composites Science and Technology 49 (1993): 277-290.

20. I. G. Farben, Verfahren zur Herstellung von Polyurethanen bzw. Polyharnstoffen, in P. Deutsches Reich Reichspatentamt, ed., Espacenet, EU Intellectual Property Office (Germany, 1942). 21. A. J. Rojas, J. H. Marciano, R. J. Williams, Rigid polyurethane foams: A model of the foaming process, Polymer Engineering \& Science 22 (1982): 840-844.

22. J. H. Marciano, M. M. Reboredo, A. J. Rojas, R. J. J. Williams, Integral-skin polyurethane foams, Polymer Engineering and Science 26 (1986): 717-724.

23. V. K. Gupta, D. V. Khakhar, Formation of integral skin polyurethane foams, Polymer Engineering and Science 39 (1999): 164-176. 
24. M. Modesti, V. Adriani, F. Simioni, Chemical and physical blowing agents in structural polyurethane foams: Simulation and characterization, Polymer Engineering and Science 40 (2000): 2046-2057.

25. X. D. Zhang, C. W. Macosko, H. T. Davis, A. D. Nikolov, D. T. Wasan, Role of silicone surfactant in flexible polyurethane foam, Journal of Colloid and Interface Science 215 (1999): 270-279.

26. J. R. Youn, H. Park, Bubble growth in reaction injection molded parts foamed by ultrasonic excitation, Polymer Engineering and Science 39 (1999): 457-468.

27. G. J. Price, E. J. Lenz, C. W. G. Ansell, The effect of high intensity ultrasound on the synthesis of some polyurethanes, European Polymer Journal 38 (2002): 1531-1536.

28. Y. Chen, H. Li, Effect of ultrasound on the viscoelasticity and rheology of polystyrene extruded through a slit die, Journal of Applied Polymer Science 100 (2006): 2907-2911.

29. J. D. Floros, H. Liang, Acoustically assisted diffusion through membranes and biomaterials, Food Technology 48 (1994): 79-84.

30. A. Hu, J. Zheng, T. Qiu, Industrial experiments for the application of ultrasound on scale control in the Chinese sugar industry, Ultrasonics Sonochemistry 13 (2006): 329-333.

31. C. Torres-Sánchez, Generation of heterogeneous cellular structures by sonication, Ph.D. thesis. Heriot-Watt University (2008).

32. A. Kim, M. A. Hasan, S. H. Nahm, S. S. Cho, Evaluation of compressive mechanical properties of Al-foams using electrical conductivity, Composite Structures 71 (2005): 191-198.

33. Y. Feng, H. W. Zheng, Z. G. Zhu, F. Q. Zu, The microstructure and electrical conductivity of aluminum alloy foams, Materials Chemistry and Physics 78 (2003): 196-201.

34. C. L. Chen, E. Lopez, P. Makaram, S. Selvarasah, A. Busnaina, Y. J. Jung, S. Muftu, M. R. Dokmeci, Fabrication and evaluation of carbon nanotube-parylene functional composite-films, Transducers '07 \& Eurosensors Xxi, Digest of Technical Papers, Vols 1 and 2 (2007): U514-U515.

35. C. Torres-Sánchez, J. Corney, Effects of ultrasound on polymeric foam porosity, Ultrasonics Sonochemistry 15 (2008): 408-415.

36. S. H. Lipshitz, C. W. Macosko, Rheological changes during a urethane network polymerization, Polymer Engineering \& Science 16 (1976): 803-810. 


\section{Figure captions}

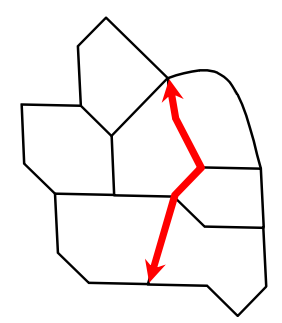

(a) Initial stages of polymer foaming

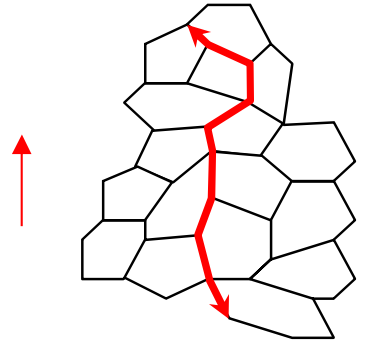

(b) Later stages: reticulated foam

Figure 1: Cross-linking process monitored by resistivity

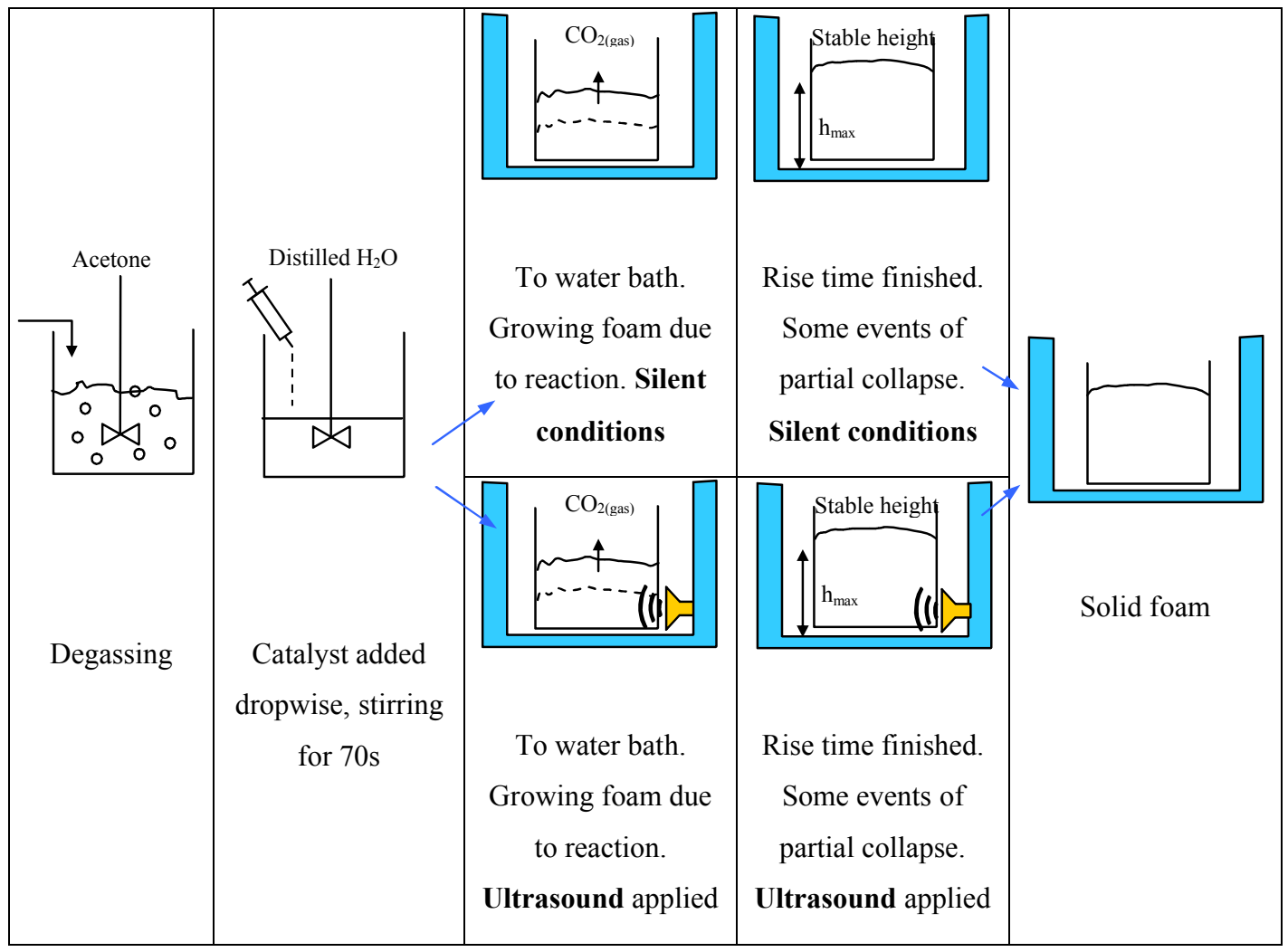

Figure 2: Foam formation in the lab 


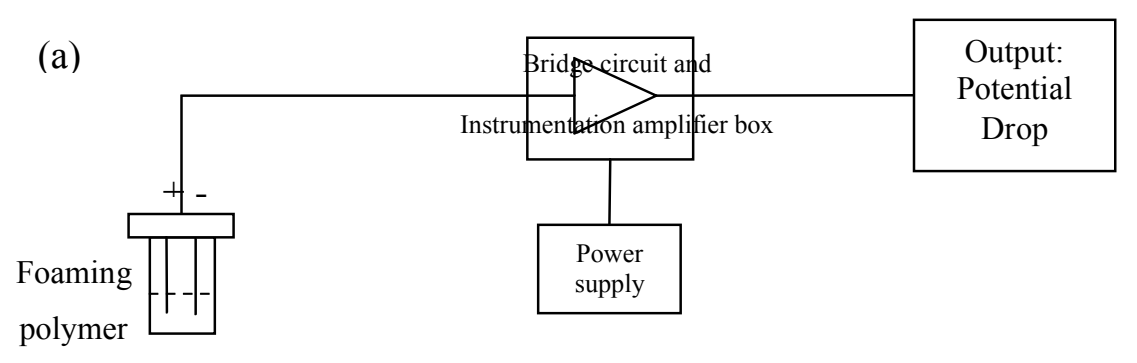

(b)

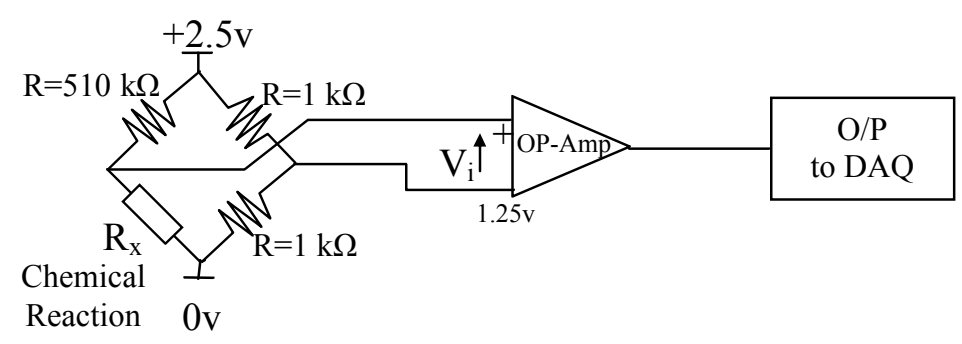

Figure 3: (a) Circuit Diagram of the apparatus used in two-probe resistivity measurement method; (b) Electronic diagram of the logic used

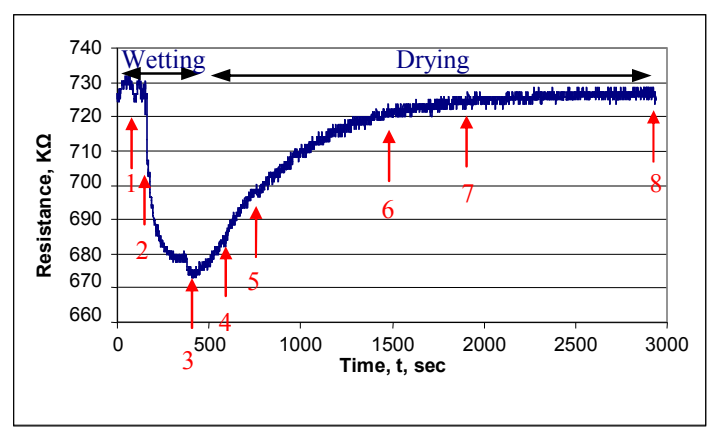

(a)

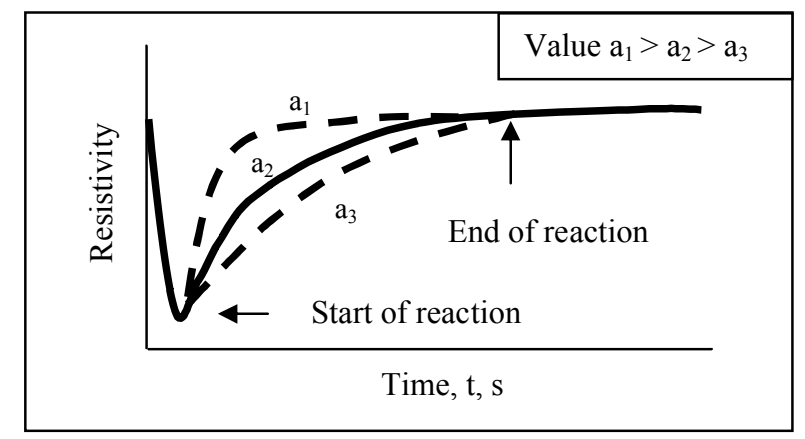

(b)

Figure 4: (a) Electrical resistance data collected by DAQ; (b) Corresponding, Logarithmic phase of the resistivity with different slope constant 'a' 


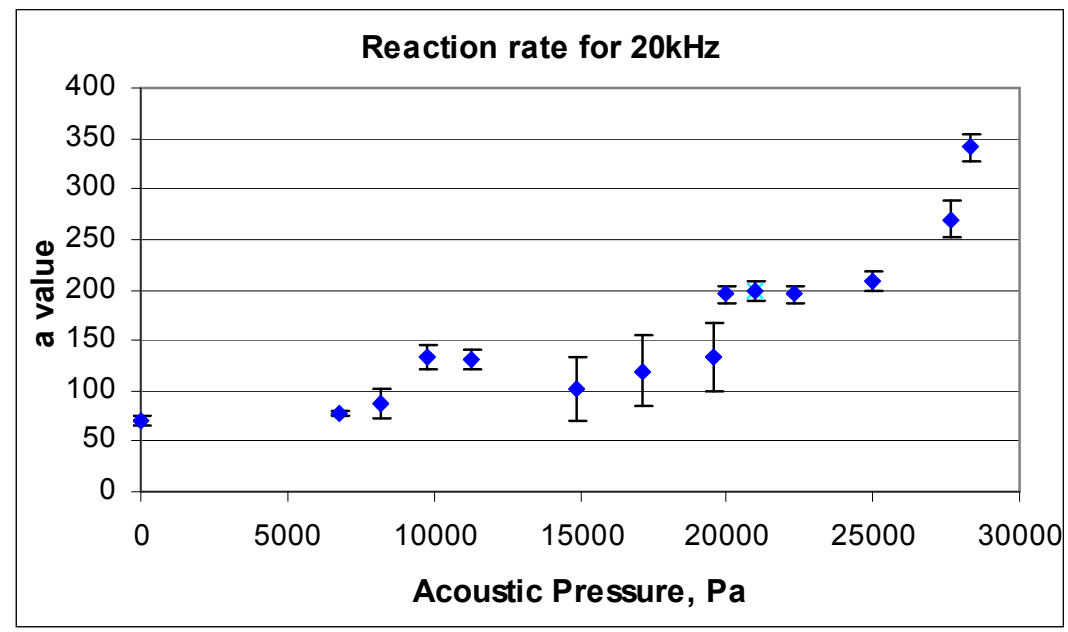

Figure 5: Slope constant 'a' value for different samples irradiated at $20 \mathrm{kHz}$

\section{Tables}

\begin{tabular}{|l|l|}
\hline Stages & Events \\
\hline Cream & Bubble generation, nucleation \\
\hline Rising & Foam growth \\
\hline Packing & Formation of the bubble network and cell window stabilisation \\
\hline Gelation & Polymer stiffening and final pore size established \\
\hline Solidification & Final curing \\
\hline
\end{tabular}

Table 1: Polymer processing, different stages of formation 


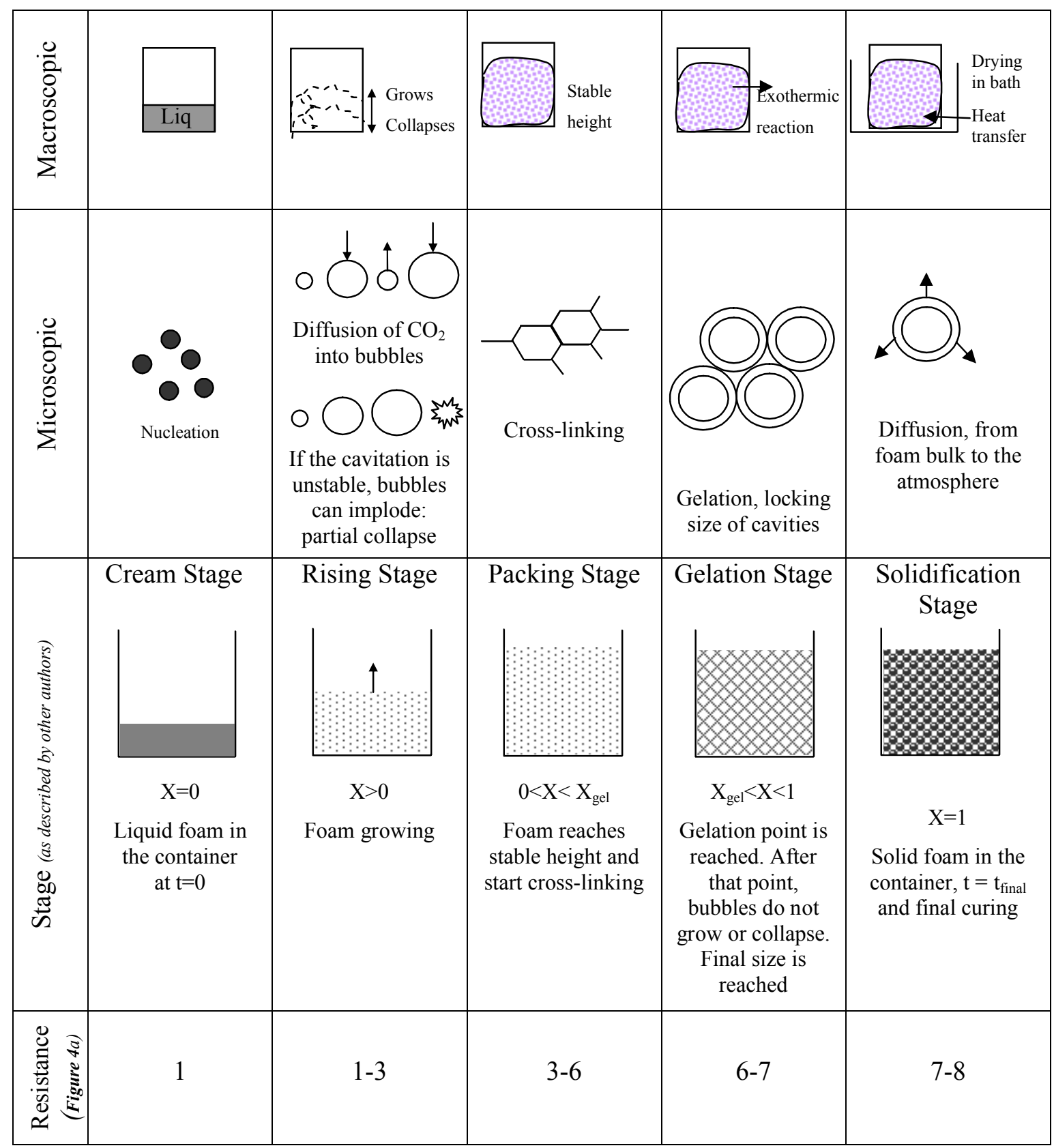

Table 2: Compilation of events during foam formation 